\title{
Energy-Harvesting Irregular Repetition Slotted ALOHA with Unit-Sized Battery
}

\author{
Umut Demirhan \\ School of ECEE, Arizona State University, Tempe, AZ \\ Email: udemirha@asu.edu
}

\author{
Tolga M. Duman \\ EEE Dept., Bilkent University, Ankara, Turkey \\ Email: duman@ee.bilkent.edu.tr
}

\begin{abstract}
We propose an irregular repetition slotted ALOHA (IRSA) based uncoordinated random access scheme for energy harvesting (EH) nodes. Specifically, we consider the case in which each user has a unit-sized battery that is recharged with energy harvested from the environment in a probabilistic manner. We analyze this scheme by deriving asymptotic throughput expressions, and obtain optimized probability distributions for the number of packet replicas for each user. We demonstrate that for the case of IRSA with EH nodes, these optimized distributions perform considerably better than those of slotted ALOHA (SA), contention resolution diversity slotted ALOHA (CRDSA) and IRSA, which do not take into account the EH process, for both asymptotic and finite frame length scenarios.
\end{abstract}

Index Terms-Slotted ALOHA, energy harvesting, machine-tomachine (M2M) communications.

\section{INTRODUCTION}

Recent developments on successive interference cancellation (SIC) enabled random access schemes have shown very promising results in terms of increasing the system throughput and providing a stable network to many users, particularly, for the emerging machine-to-machine (M2M) communications in 5G. Since it is of interest to devise systems that operate for a very long time, such sensing and communication nodes should be equipped with energy harvesting (EH) capabilities to work without the need for battery replacement. Therefore, it is essential to study ALOHA schemes providing uncoordinated random access (RA) with high throughputs using EH nodes.

Originating from diversity ALOHA [2], which utilizes time and frequency diversity for transmitting multiple copies of users' packets, contention resolution diversity slotted ALOHA (CRDSA) [3] considers grouping a number of slots as frames in which each user sends two copies of its packets referred to as replicas. SIC among slots may be adopted to resolve the collisions given the received signals in the entire frame. That is, a collision may be resolved by subtracting a decoded packet from the collision that includes its replica, resulting in a maximum throughput of $T \simeq 0.55$, which is defined as successfully resolved packets per slot [3].

After representing the SIC process explicitly on a bipartite graph, Liva proposes to vary the number of copies according to a probability distribution resulting in irregular repetition slotted ALOHA (IRSA), and describes an iterative process to analyze the asymptotic performance of the system for a fixed repetition distribution [4]. He also shows that it is possible to

This work is based on Umut Demirhan's M.S. Thesis [1] completed at Bilkent University. optimize the repetition distribution, resulting in IRSA schemes with a maximum asymptotic throughput close to the maximum value of 1 on the collision channel. As an extension, it is also shown that frame and slot asynchronous approaches with SIC provide significant improvements as well [5]-[7].

Study of MAC protocols including ALOHA variants with EH nodes are available in many existing papers. In [8], the authors examine the stability of slotted ALOHA in an EH environment. In [9], a scheme is proposed to optimize the sum throughput of slotted ALOHA in which dynamically selected transmission power is adopted depending on the EH rate and battery size. Various MAC protocols including frame ALOHA, frame slotted ALOHA (FSA) and dynamic FSA for an energy harvesting system are studied in [10]. The authors in [11] consider energy harvesting aware dynamic frame slotted ALOHA for M2M networks. Moreover, [12] examines reservation dynamic frame slotted ALOHA for M2M networks. On the other hand, there is no existing work proposing a scheme based on CRDSA or IRSA providing high throughputs with EH nodes. Given the drastically increasing number of machine type devices and their expected lifetime of operation, throughput maximization on uncoordinated RA schemes with EH nodes has a tremendous potential. Therefore, our interest in this paper is to investigate the applicability of IRSA for an $\mathrm{EH}$ system for the first time in the literature.

We propose a modified IRSA scheme accommodating EH nodes named as energy-harvesting irregular repetition slotted ALOHA (EH-IRSA). In the proposed scheme, sporadically activated users with $\mathrm{EH}$ capabilities are equipped with a unitsized battery that can provide energy for one packet transmission. Users draw the number of replicas for their packets from a probability distribution and select specific time slots to send them across a frame, similar to IRSA. On the other hand, if a user has no energy in its selected slot, transmission cannot take place, hence the allocated slot is skipped. In our model, we assume that an empty battery is filled with a certain probability in each slot, independently of the other slots and users. We analyze the asymptotic throughput of the system for a fixed EH rate, which is defined as the expected number of energy arrivals in a frame, and we show that finite MAC frame length throughput performances conform with the theoretical (asymptotic) results. We utilize the derived results to find the optimal degree distributions for packet replicas via differential evolution for different EH rates. Our numerical results demonstrate the superiority of the proposed solutions 
both asymptotically and through extensive finite frame length simulations for the case with EH nodes over the distributions of SA, CRDSA and the optimized distributions of IRSA, which do not take into account the EH process.

The paper is organized as follows. In Section II, the system model is given. The proposed EH-IRSA scheme is described and its convergence analysis is conducted in Section III. In Section IV, several optimized packet replica distributions are obtained and their performances are compared with those of CRDSA and IRSA (optimized without the EH considerations), and superiority of the proposed solution within the EH framework is demonstrated. Conclusions are provided in Section V.

\section{SySTEM MODEL}

We consider a slotted ALOHA system in which the time slots are grouped as MAC frames (simply referred to as frames). Each frame consists of $N$ equal length slots. The number of total users that are sporadically activated (that send messages to a common receiver) is denoted by $M_{t}$. The users are synchronized across the time slots and frames. Each user is activated with a probability $\pi$ for a given frame independently of activations in other frames and other users. The number of active users in a frame is $M_{a}$. Expected channel load $G$ is defined as the expected number of active users per slot given by

$$
G=\frac{\mathbb{E}\left[M_{a}\right]}{N}=\frac{\pi M_{t}}{N} .
$$

The transmitting nodes are capable of harvesting energy from a renewable energy source and each is equipped with a battery of capacity $\delta$. We assume that transmission of each packet replica consumes $\delta$ energy. The batteries of the users are fully recharged with probability $p_{c}$, independently, in any given time slot ${ }^{1}$. The probability of no energy arrival in a particular slot is $p_{n c}=1-p_{c}$. The arriving energy is lost if the battery is full. We simply take $\delta=1$ without any loss of generality.

Let $E_{s}$ denote the amount of energy in the battery at the beginning of the $s$-th slot in a frame. If there is a transmission attempt in that slot, $E_{s+1}=0$, independent of the energy arrival and success of the transmission. If there is no transmission attempt in that slot,

$$
E_{s+1}= \begin{cases}1 & \text { with probability } p_{c} \\ E_{s} & \text { with probability } p_{n c} .\end{cases}
$$

We use the common channel model utilized in the analysis of RA schemes as in [3], [4], namely, the collision channel, and make similar assumptions throughout this paper. Particularly, we assume that the receiver is able to recognize slots with a single replica, collisions and no transmission. Slots with a single replica are always successfully decoded. On the other hand, collisions are considered as non-resolvable, i.e., no information can be obtained from the collision in the absence of any additional knowledge. Each packet carries the

\footnotetext{
${ }^{1}$ The adopted energy harvesting and battery models are common in the communications literature as an abstraction and simplification of more general systems (e.g. [13], [14]).
}

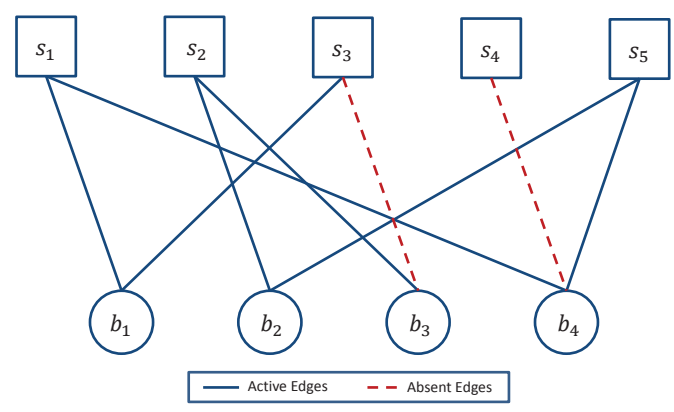

Fig. 1. An illustration of the proposed scheme with 4 users and 5 slots on a bipartite graph. User nodes (circles) represent users and sum nodes (squares) represent slots. Edges connect the users to their pre-determined slots.

information on which slots its copies are sent and that part of the packet is decoded independently of the whole packet that enables SIC to be employed at the receiver ${ }^{2}$.

\section{ENERGY-HARVESTING IRSA}

In this section, we describe the proposed scheme and analyze its throughput. First, let us briefly review the original IRSA [4]. In IRSA, each user sends $k$ replicas of its packets with probability $\Lambda_{k}$ in any given frame. The probability mass function (PMF) of the number of replicas sent is represented by the polynomial $\Lambda(x)=\sum_{k=0}^{k_{\max }} \Lambda_{k} x^{k}$, which will be referred to as the repetition distribution (RD). The slots in which the replicas are sent are selected uniformly in each frame. The receiver resolves the packets through an iterative process implementing SIC by removing the other copies in different slots of successfully decoded packets. This process can be represented on a bipartite graph and it is similar to decoding process of low density parity check (LDPC) codes, which leads to the optimization of the repetition distribution through the density evolution techniques [4].

In the proposed EH-IRSA, the same protocol is adopted along with a modification taking into account the energy state of the battery. A user transmits its replicas during its predetermined slots only if there is energy stored in its battery, and does not transmit otherwise. In this scheme, although a user may have a certain number of replicas to send, some of these are clearly not transmitted. If a node uses an RD of $\Lambda(x)$, due to the energy constraints, it will have a different distribution $\tilde{\Lambda}(x)$ corresponding to the probability distribution of the replicas actually being transmitted, which is referred to as the effective repetition distribution (ERD). Replicas, which are not sent due to lack of energy, are called as absent, and the rest that are successfully sent are referred to as active. In Fig. 1, an example of this scheme is depicted on a bipartite graph. In this example, the number of replicas users try to send are $\{2,2,2,3\}$. However, they effectively send $\{2,2,1,2\}$ replicas. If this example was reflecting the expected number of packets and energy arrivals, the RD would be $\Lambda_{e}(x)=$ $\frac{3}{4} x^{2}+\frac{1}{4} x^{3}$ resulting in an ERD of $\tilde{\Lambda}_{e}(x)=\frac{1}{4} x+\frac{3}{4} x^{2}$.

\footnotetext{
${ }^{2}$ To eliminate the probable signaling burden, the random number generators of the users may be synchronized with the receiver.
} 


\section{A. Convergence Analysis}

We are interested in the asymptotic throughput behavior for a load level $G$ and an EH rate $\alpha$, which is defined as the expected number of energy arrivals in a frame given by $\alpha=$ $N p_{c}$. We let $M, N \rightarrow \infty$ and $p_{c} \rightarrow 0$ while keeping the EH rate $\alpha=N p_{c}$ a constant, and analyze the SIC process from a probabilistic perspective. In other words, we analyze the effects of different energy arrival rates asymptotically. The density evolution analysis is similar to that of IRSA in [4], hence the details are omitted.

We define user node (UN) and sum node (SN) distributions from the edge perspective as

$$
\lambda(x) \triangleq \sum_{l} \lambda_{l} x^{l-1}, \quad \rho(x) \triangleq \sum_{l} \rho_{l} x^{l-1}
$$

where $\lambda_{l}$ and $\rho_{l}$ correspond to the probability of an edge being connected to a degree- $l \mathrm{UN}$ and a degree- $l \mathrm{SN}$, respectively. Let $q_{i}$ and $p_{i}$ denote the probability of an edge not being revealed after the $i$-th iteration from a $\mathrm{UN}$ and an $\mathrm{SN}$, respectively. These quantities can be calculated by iterating through $q_{i}=\lambda\left(p_{i-1}\right)$ and $p_{i}=1-\rho\left(1-q_{i}\right)$.

Let us now define the packet loss ratio (PLR) as the ratio between the number of active users whose packets are not resolved and the number of active users, which is an important metric in the evaluation of the system performance. We can obtain the PLR by converting the edge perspective probability to the node perspective probability using PLR $=\Lambda\left(p_{m}\right)$ for a selected arbitrarily large number of SIC iterations, $m$, representing $m \rightarrow \infty$.

The system throughput is defined as $T=G(1-\mathrm{PLR})^{3}$. By utilizing a differential evolution based optimization algorithm [15], we obtain RDs that provide the maximum channel throughput $T^{*}$. We also consider the classical approach that maximizes the offered channel load $G$ whilst achieving an arbitrarily chosen target PLR that is close to 0 in our numerical results ${ }^{4}$.

To calculate the resulting PLR, we need to obtain $\rho(x)$ and $\lambda(x)$ polynomials, which are derived in [4] as $\lambda(x)=\frac{\Lambda^{\prime}(x)}{\Lambda^{\prime}(1)}$ and $\rho(x)=e^{-G \Lambda^{\prime}(1)(1-x)}$. Differently from [4], we utilize the ERDs instead of the RDs in the density evolution analysis of the decoding process. To clarify, we only use the active packet replicas, i.e., the absent packet replicas have no effect on the SIC process. We further assume that the receiver is able to obtain the knowledge of active and absent edges and it can figure out when a replica is skipped due to lack of energy ${ }^{5}$.

\footnotetext{
${ }^{3}$ For the simulations, $G_{a}=\frac{M_{a}}{N}$ and PLR are random variables, and throughput is the expectation of the equation across the frames, i.e., $T=$ $E\left[G_{a}(1-\mathrm{PLR})\right]$.

${ }^{4}$ This is the conventional approach where a search is done over RDs to find the maximum channel load (which is almost equal to the throughput for low PLRs) that provides a target PLR arbitrarily close to 0 . However, our main approach is different since the case in which a user may not be able to send a replica because of no energy arrival before the last selected slot may have a non-negligible probability preventing $P_{l}$ from going to zero.

${ }^{5}$ Determining absent edges is a detection problem that should be considered jointly with the code utilized in the system. It is beyond the scope of this paper.
}

We need to compute the ERD corresponding to a given $\mathrm{RD}$ for the analysis of the SIC process to utilize in the edge perspective probability calculations $\rho(x)$ and $\lambda(x)$. Denoting the ERD as $\tilde{\Lambda}(x)=\sum_{k=0}^{k_{\max }} \tilde{\Lambda}_{k} x^{k}$, we simply change $\Lambda(x)$ to $\tilde{\Lambda}(x)$ in the equations of $\rho$ and $\lambda$ by making an approximation that active replicas are uniformly distributed over each frame. Then we obtain $\lambda(x)=\frac{\tilde{\Lambda}^{\prime}(x)}{\tilde{\Lambda}^{\prime}(1)}$ and $\rho(x)=e^{-G \tilde{\Lambda}^{\prime}(1)(1-x)}$.

To clarify the approximation further, we note that, in IRSA, the distribution of the number of packets received on a slot is a binomial random variable because of the uniformity of the selected slots for sending packets. On the other hand, in EHIRSA, this is not the case. For instance, clearly the first packet has a different probability than the others due to the sporadic activity of users. Users charge their batteries while they are not active which in turn changes the probability of successfully sending the first packet in a frame. Nevertheless, we assume that the distribution of active replicas is approximately uniform to make the analysis tractable and calculable. We verify the accuracy of this approximation in the numerical results section by comparing the asymptotic results with the finite frame length simulations.

\section{B. Effective Repetition Distributions}

In this subsection, we compute the ERD for a given RD and EH rate asymptotically as $N, M \rightarrow \infty$ with $\alpha=N p_{c}$ being a constant. We define $\Phi_{l}^{k}$ as the probability of having $l$ active replicas out of a total of $k$ active and absent replicas, i.e., selected slots for the replica transmissions. We define $\Phi^{k}(x)$, which represents the PMF of the number of active replicas when the user selects $k$ replicas to be transmitted in a frame. The resulting ERD and $\Phi^{k}(x)$ can be written as

$$
\tilde{\Lambda}(x)=\sum_{k=0}^{k_{\max }} \Lambda_{k} \Phi^{k}(x), \quad \Phi^{k}(x)=\sum_{l=0}^{k} \Phi_{l}^{k} x^{l},
$$

which simply results in $\tilde{\Lambda}_{l}=\sum_{k=0}^{k_{\max }} \Lambda_{k} \Phi_{l}^{k}$.

Due to the sporadic activity of users, slots after the last transmission trial should be considered to calculate the probability of a user's battery being recharged or not at the beginning of a frame. Let $\operatorname{Pr}\left\{E_{0}=0\right\}$ denote the probability of not having energy and $\operatorname{Pr}\left\{E_{0}=1\right\}=1-\operatorname{Pr}\left\{E_{0}=0\right\}$ be the probability of the battery being full at the beginning of a frame. We split $\Phi_{l}^{k}$ into two parts based on its battery state at the beginning of a frame as

$$
\Phi_{l}^{k}=\operatorname{Pr}\left\{E_{0}=1\right\} \phi_{l}^{k}+\operatorname{Pr}\left\{E_{0}=0\right\} \bar{\phi}_{l}^{k}
$$

where $\phi_{l}^{k}$ is the probability of $l$ active replicas over $k$ replicas when the first replica is successfully transmitted using the energy at the beginning of the frame, and similarly, $\bar{\phi}_{l}^{k}$ is the probability of successful transmission of $l$ replicas over $k$ replicas when the user has no energy at the beginning of the frame.

To derive $\phi_{l}^{k}$ and $\bar{\phi}_{l}^{k}$, we average across the possible selections of $k$ slots and $l$ successful transmissions by using the separations (referred to as distances) among the selected slots denoted by the vector $\boldsymbol{d}=\left\{d_{1}, d_{2}, \ldots, d_{k}\right\}$. Note that $d_{1}$ 


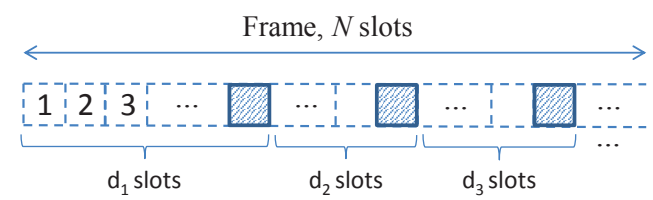

Fig. 2. Distances in a frame.

is the position of the first selected slot, while $d_{i}$ for $i>1$ is the slot distance between the $(i-1)$-th and $i$-th selected slots. These distances represent the number of slots for energy arrivals before a transmission as shown in Fig. 2 .

We use $|\cdot|_{1}$ to denote the $l_{1}$-norm, and $[k]$ as the set of integers from 1 to $k$. For every $k \in \mathbb{Z}_{+}$and $l \in \mathbb{N}$ with $l \leq k$, $D=\left\{\boldsymbol{d} \in \mathbb{Z}_{+}^{k}:|\boldsymbol{d}|_{1} \leq N\right\}$ is the set of all possible distance selections, $B_{l}^{k}=\left\{\boldsymbol{b} \in[k]^{l}: b_{i} \neq b_{j} \forall i, j \in[l]\right.$ with $\left.i \neq j\right\}$ is the set of all possible selection of indices of $l$ active replicas. With this notation, we can write

$$
\bar{\phi}_{l}^{k}=\lim _{\substack{N \rightarrow \infty \\ p_{c} \rightarrow 0 \\ N p_{c}=\alpha}} \sum_{\boldsymbol{b} \in B_{l}^{k}} \sum_{\boldsymbol{d} \in D} P(\boldsymbol{d}) \prod_{j \in \boldsymbol{b}}\left(1-p_{n c}^{d_{j}}\right) \prod_{i \in[k] \backslash \boldsymbol{b}}\left(p_{n c}^{d_{i}}\right) .
$$

The first summation in (3) is over all the possible $l$ active slot selections out of $k$ total slots. The set $D$ is symmetric for each dimension, i.e., the $i$-th and $j$-th elements for any $i, j$ can be interchanged over $D$ without changing $D$. Therefore, for any $\boldsymbol{b} \in B_{l}^{k}$, the inner summation is the same. Hence, we obtain

$$
\bar{\phi}_{l}^{k}=\lim _{\substack{N \rightarrow \infty \\
p_{c} \rightarrow 0 \\
N p_{c}=\alpha}} \frac{\left(\begin{array}{l}
k \\
l
\end{array}\right)}{\left(\begin{array}{l}
N \\
k
\end{array}\right)} \sum_{\boldsymbol{d} \in D} p_{n c}^{\sum_{i=l+1}^{k} d_{i}} \prod_{j=1}^{l}\left(1-p_{n c}^{d_{j}}\right) .
$$

We modify (4) by further exploiting this symmetry of $D$ and change $d_{i}$ and $d_{j}$ terms for some $i, j$ inside the summation over $D$ and write

$$
\bar{\phi}_{l}^{k}=\lim _{\substack{N \rightarrow \infty \\
p_{c} \rightarrow 0 \\
N p_{c}=\alpha}} \frac{\left(\begin{array}{l}
k \\
l
\end{array}\right)}{\left(\begin{array}{c}
N \\
k
\end{array}\right)} \sum_{\boldsymbol{d} \in D} p_{n c}^{\sum_{i=l+1}^{k} d_{i}} \sum_{r=0}^{l}(-1)^{r}\left(\begin{array}{l}
l \\
r
\end{array}\right) p_{n c}^{\sum_{j=1}^{r} d_{j}}
$$

Defining

$$
f(k, r)=\lim _{\substack{N \rightarrow \infty \\
p_{c} \rightarrow 0 \\
N p_{c}=\alpha}} \frac{1}{\left(\begin{array}{c}
N \\
k
\end{array}\right)} \sum_{\boldsymbol{d} \in D} p_{n c} \sum_{i=1}^{r} d_{i},
$$

and by using (5) and (6), we obtain

$$
\bar{\phi}_{l}^{k}=\left(\begin{array}{l}
k \\
l
\end{array}\right) \sum_{r=0}^{l}\left(\begin{array}{l}
l \\
r
\end{array}\right)(-1)^{r} f(k, k-l+r) .
$$

We can numerically calculate $f(k, r)$ for all possible $k$ and $r$ values, and we can compute any $\bar{\phi}_{l}^{k}$ term from a given RD. Moreover, the complexity of the numerical calculations can be decreased with the following recursive equations

$$
\begin{gathered}
-\frac{d f(k, r)}{d \alpha} \frac{k+1}{r}=f(k+1, r+1), \\
\left(\frac{d f(k, r)}{d \alpha}+f(k, r)\right) \frac{k+1}{k+1-r}=f(k+1, r),
\end{gathered}
$$

$$
f(1,1)=\frac{1-e^{-a}}{\alpha}, \quad k, r \geq 1,
$$

whose proofs can be found in [1].

For the case with full battery at the beginning of a frame, we consider $\tilde{k}=k-1$ and $\tilde{l}=l-1$, and ignore the first replica that is certainly transmitted since battery is full at the start of the frame. Thus, in a complementary manner to (4)-(7), we obtain

$$
\begin{aligned}
\phi_{l}^{k} & =\lim _{\substack{N \rightarrow \infty \\
p_{c} \rightarrow 0 \\
N p_{c}=\alpha}} \frac{\left(\begin{array}{c}
\tilde{k} \\
\tilde{l}
\end{array}\right)}{\left(\begin{array}{c}
N \\
k
\end{array}\right)} \sum_{\boldsymbol{d} \in D} p_{n c}^{\sum_{i=l+1}^{k} d_{i}} \prod_{j=2}^{l}\left(1-p_{n c}^{d_{j}}\right), \\
& =\left(\begin{array}{c}
\tilde{k} \\
\tilde{l}
\end{array}\right) \sum_{r=0}^{\tilde{l}}\left(\begin{array}{l}
\tilde{l} \\
r
\end{array}\right)(-1)^{r} f(k, k-l+r) .
\end{aligned}
$$

To sum up, we can compute the $\phi_{l}^{k}$ and $\bar{\phi}_{l}^{k}$ terms corresponding to any given $\mathrm{RD}$ and any $\mathrm{EH}$ rate $\alpha$, which can be utilized to obtain the asymptotic throughput performance of the system.

In our analysis, we also need the probabilities $\operatorname{Pr}\left\{E_{0}=0\right\}$ and $\operatorname{Pr}\left\{E_{0}=1\right\}$, which are computed under the same asymptotic assumptions. These probabilities depend on two different variables: the last slot that a user tries to send a replica and the number of frames between two consecutive frames that the user is active. We denote the average probability of not being charged after the last trial of sending the replica in that frame as $P_{\text {slots }}^{\prime}$ and the average probability of being not charged during the frames that the user is not active as $P_{\text {frames }}^{\prime}$. We write

$$
\operatorname{Pr}\left\{E_{0}=0\right\}=P_{\text {slots }}^{\prime} P_{\text {frames }}^{\prime},
$$

as these two events are independent. We form $P_{\text {slots }}^{\prime}$ as

$$
P_{\text {slots }}^{\prime}=\lim _{\substack{N \rightarrow \infty \\
p_{c} \rightarrow 0 \\
N p_{c}=\alpha}} \sum_{k=1}^{k_{\max }} \Lambda_{k} \sum_{i=1}^{N-k+1}\left(\begin{array}{c}
N-i \\
k-1
\end{array}\right) p_{n c}^{i}
$$

which is the average probability of the battery not being charged after the last trial of transmission. We note that the inner summation is equivalent to $f(k, 1)$, and simplify the expression as

$$
P_{\text {slots }}^{\prime}=\sum_{k=1}^{k_{\max }} \Lambda_{k} f(k, 1) .
$$

The effect of the frames between the consecutive frames that a user is active can be expressed as the probability of no energy arrival in those frames, i.e.,

$$
\begin{aligned}
P_{\text {frames }}^{\prime} & =\lim _{\substack{N \rightarrow \infty \\
p_{c} \rightarrow 0 \\
N p_{c}=\alpha}} \pi p_{n c}^{0}+\pi(1-\pi) p_{n c}^{N}+\pi(1-\pi)^{2} p_{n c}^{2 N}+\ldots \\
& =\frac{\pi}{1-(1-\pi) e^{-\alpha}}
\end{aligned}
$$

Finally, by combining (12), (14) and (15), we obtain

$$
\operatorname{Pr}\left\{E_{0}=0\right\}=\frac{\pi}{1-(1-\pi) e^{-\alpha}} \sum_{k=1}^{k_{\max }} \Lambda_{k} f(k, 1) .
$$


TABLE I

ASYMPTOTIC PERFORMANCES OF DISTRIBUTIONS FOR $\alpha=5$.

\begin{tabular}{|c|c|c|c|}
\hline \hline $\boldsymbol{\alpha}$ & $\boldsymbol{\pi}$ & Distribution, $\boldsymbol{\Lambda}(\boldsymbol{x})$ & $\boldsymbol{T}^{*}$ \\
\hline \hline \multirow{3}{*}{5} & \multirow{3}{*}{0.1} & $\Lambda_{1}(x)=0.25 x^{3}+0.13 x^{7}+0.62 x^{8}$ & 0.8701 \\
\cline { 3 - 4 } & & $\Lambda_{L}(x)=0.5 x^{2}+0.28 x^{3}+0.22 x^{8}$ & 0.5791 \\
\cline { 3 - 4 } & \multirow{3}{*}{1} & $\Lambda_{C}(x)=x^{2}$ & 0.4633 \\
\cline { 3 - 4 } & \multirow{2}{*}{1} & $\Lambda_{2}(x)=0.02 x^{4}+0.02 x^{5}+0.34 x^{7}+0.62 x^{8}$ & 0.8682 \\
\cline { 3 - 4 } & & $\Lambda_{L}(x)$ & 0.5521 \\
\cline { 3 - 4 } & & $\Lambda_{C}(x)$ & 0.4542 \\
\hline
\end{tabular}

\section{NUMERICAL RESULTS}

We now present several numerical examples to illustrate the results of our analysis along with the simulations of the EHIRSA scheme. For fixed $\alpha$ and $\pi$ values, we search for RDs providing the largest $T^{*}$ values via differential evolution using the proposed convergence analysis. We limit the maximum repetition degree $k_{\max }$ to 8 in our design. The resulting maximum asymptotic throughputs of the optimized distributions for $\alpha=5$ and $\pi=0.1,1$ are presented in Table I. ${ }^{6}$ For the finite length simulations, we normalize the number of total users $M_{t}$ to change the expected load $G$ while keeping the activity probability $\pi$ and number of slots $N$ the same.

Figs. 3 and 4 depict the resulting throughputs as a function of $G$. In Fig. 3 (with $\pi=0.1$ ), we observe that the maximum throughput provided by $\Lambda_{L}(x)$ is about 0.58 while the newly optimized distributions can offer a maximum asymptotic throughput of 0.87 , which is a substantial gain. In Fig. 4 depicting the optimization results with $\pi=1$, we make similar observations, namely, the expected throughputs are significantly higher with the newly optimized distributions. Also note that finite size simulation results conform with the results obtained via the asymptotic analysis, hence they verify the accuracy of the approximation used. The classical waterfall effect of the optimization results with density evolution in the PLR graphs is also observed.

To illustrate the system performance with different energy harvesting rates, we provide Fig. 5, which depicts the maximum throughputs of the newly optimized distributions with all active users $(\pi=1)$ as a function of $\alpha$. The results show that by using the $\mathrm{EH}$ rate in the optimization process we can achieve higher throughputs compared to other distributions, namely, the distribution of slotted ALOHA, CRDSA and the 8-th order optimized distribution of IRSA [4]. Also note that as $\alpha$ increases (approaches $N$ ), the energy scarcity is alleviated, and plain IRSA optimization $\left(\Lambda_{L}\right)$ offers good results. However, our optimized distributions reach to a high throughput for considerably wide range of $\alpha$ values while $\Lambda_{L}(x)$ requires a much higher $\alpha$ value to approach such values as it can be seen from Fig. 5 .

\footnotetext{
${ }^{6} \Lambda_{L}(x)$ is the 8-th order optimized distribution of the IRSA in [4] and $\Lambda_{C}(x)$ is the regular-2 distribution of the classical CRDSA.
}
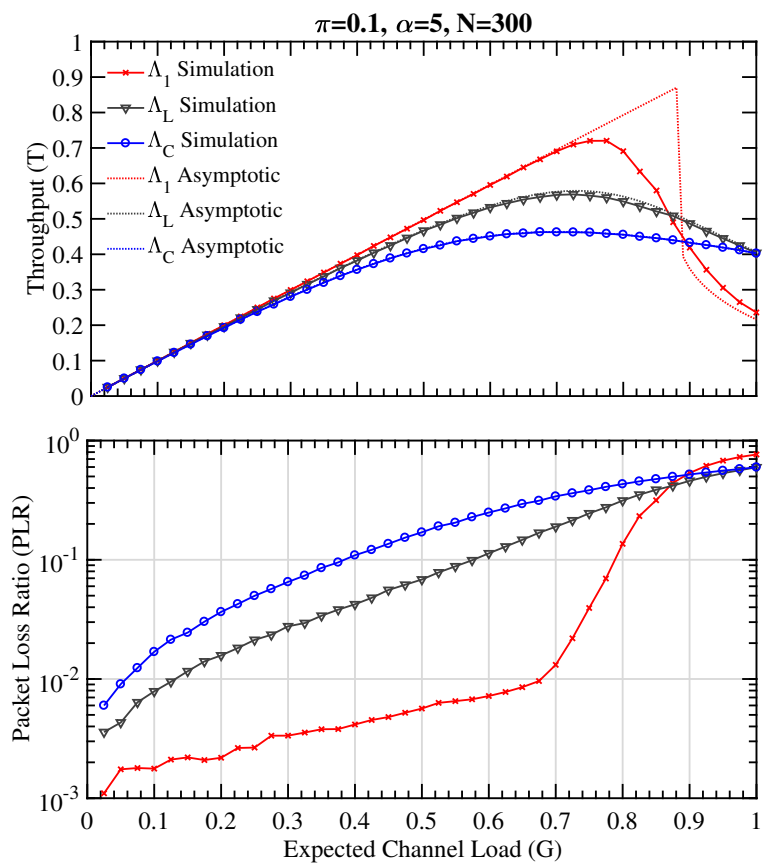

Fig. 3. The results of the simulation and analysis for energy harvesting rate of 5 , frame length of 300 and activity probability of 0.1 .
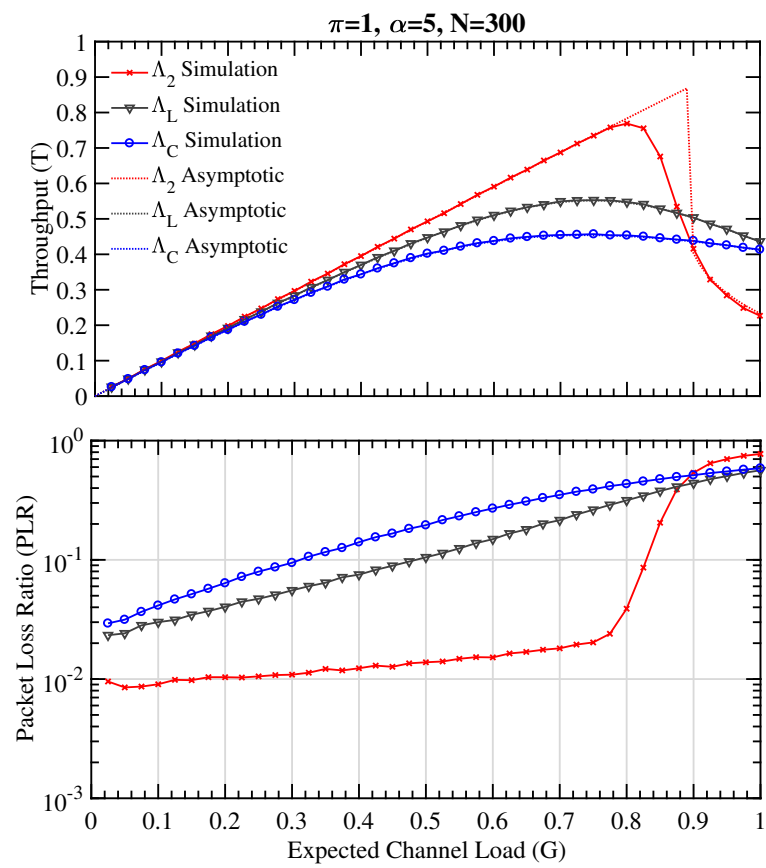

Fig. 4. The results of the simulation and analysis for energy harvesting rate of 5 , frame length of 300 and activity probability of 1 .

Fig. 6 illustrates several target PLR based throughput optimization results. Note that this PLR based optimization differs from our initial pure throughput maximization, that is, in this case, we search for the maximum channel load that provides a target PLR $=10^{-2}$. We see that the optimized distributions with both methodologies offer similar results. We conclude from Fig. 6, which shows the maximum asymptotic channel load 


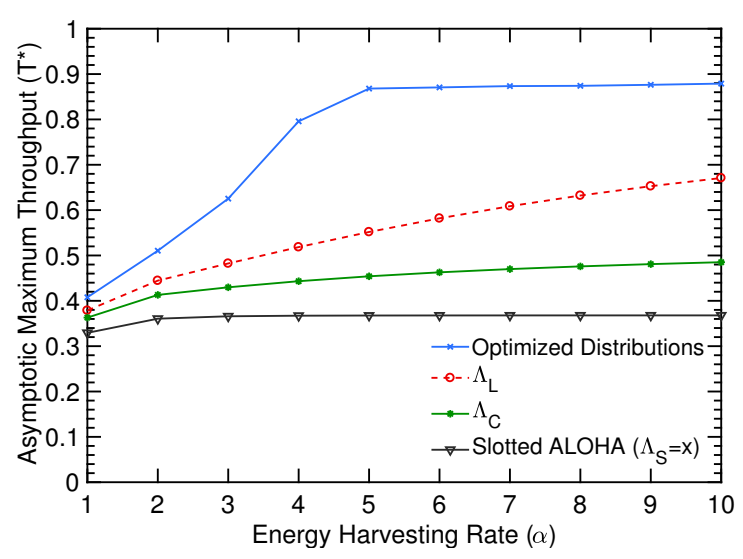

Fig. 5. Comparison of the resulting asymptotic throughputs of the distributions for different EH rates, with activity probability 1.

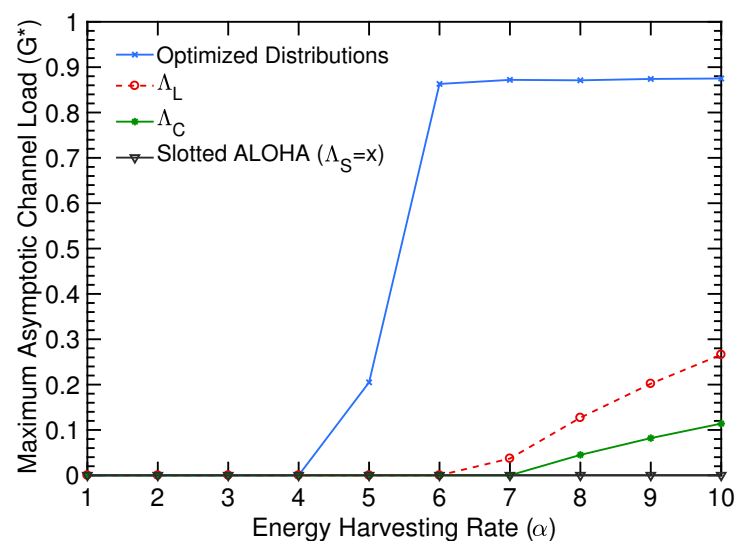

Fig. 6. Comparison of the resulting asymptotic channel loads of the distributions for different EH rates, with activity probability 1.

instead of the throughput in Fig. 5, that if the energy arrival rate is sufficiently high (e.g., $\alpha \geq 5$ for this example), the maximum throughput and channel load reaches a high value. Above a critical value of $\alpha$, the optimization of maximum channel load providing a target PLR (e.g., $10^{-2}$ ) is feasible. This critical value slightly changes with different target PLR values. Below the critical value where the target PLR is not achievable, the throughput based optimization results in the maximum degree repetition distribution $\Lambda_{\max }=x^{8}$, which is the trivial solution where the users send their replicas as soon as possible when the energy arrival is scarce ${ }^{7}$.

\section{CONCLUSIONS}

In this paper, with the aim of extending the high throughput performance of CRDSA, IRSA and variants of uncoordinated medium access schemes, we have proposed a new protocol based on IRSA for a network of EH nodes. We analyze the proposed scheme for the case in which each user has a unit-sized battery, and show that IRSA-like schemes promise networks that can handle many users with EH capabilities. The analysis we provide is simple and does not contain any critical changes on the density evolution, and can potentially be extended to more complex channel models, e.g., using the recent works on erasure channels in [16], [17] and fading channels in [18]. Furthermore, it can potentially be extended to the cases with higher battery capacity and different models on energy arrivals as well.

\section{REFERENCES}

[1] U. Demirhan, "Irregular repetition slotted ALOHA with energy harvesting nodes," M.S. Thesis, Dept. of Elect. and Electron. Eng., Bilkent University, Ankara, Turkey, 2017.

[2] G. L. Choudhury and S. S. Rappaport, "Diversity ALOHA - random access scheme for satellite communications," IEEE Trans. Commun., vol. 31, no. 3, pp. 450-457, Mar. 1983.

[3] E. Casini, R. De Gaudenzi, and O. Del Rio Herrero, "Contention resolution diversity slotted ALOHA (CRDSA): An enhanced random access scheme for satellite access packet networks," IEEE Trans. Wirel. Commun., vol. 6, no. 4, pp. 1408-1419, Apr. 2007.

[4] G. Liva, "Graph-based analysis and optimization of contention resolution diversity slotted ALOHA," IEEE Trans. Commun., vol. 59, no. 2, pp. 477-487, Feb. 2011.

[5] C. Kissling, "Performance enhancements for asynchronous random access protocols over satellite," in IEEE Int. Conf. Commun. (ICC), Kyoto, Japan, Jun. 2011.

[6] C. Stefanovic and P. Popovski, "ALOHA random access that operates as a rateless code," IEEE Trans. Commun., vol. 61, no. 11, pp. 4653-4662, Nov. 2013.

[7] R. De Gaudenzi, O. del Rio Herrero, G. Acar, and E. Garrido Barrabes, "Asynchronous contention resolution diversity ALOHA: Making CRDSA truly asynchronous," IEEE Trans. Wirel. Commun., vol. 13, no. 11, pp. 6193-6206, Nov. 2014

[8] J. Jeon and A. Ephremides, "On the stability of random multiple access with stochastic energy harvesting," IEEE J. Sel. Areas Commun., vol. 33, no. 3, pp. 571-584, Mar. 2015.

[9] M. Moradian and F. Ashtiani, "Sum throughput maximization in a slotted ALOHA network with energy harvesting nodes," in IEEE Wirel. Commun. Netw. Conf. (WCNC), Istanbul, Turkey, Apr. 2014, pp. 15851590.

[10] F. Iannello, O. Simeone, and U. Spagnolini, "Medium access control protocols for wireless sensor networks with energy harvesting," IEEE Trans. Commun., vol. 60, no. 5, pp. 1381-1389, May 2012.

[11] S. Wu, Y. Chen, K. K. Chai, F. Vazquez-Gallego, and J. Alonso-Zarate, "Analysis and performance evaluation of dynamic frame slotted-ALOHA in wireless machine-to-machine networks with energy harvesting," in IEEE Glob. Telecommun. Conf. (GLOBECOM), Austin, Texas, USA, Dec. 2014, pp. 1081-1086.

[12] F. Vazquez-Gallego, J. Alonso-Zarate, and L. Alonso, "Reservation dynamic frame slotted-ALOHA for wireless M2M networks with energy harvesting," in IEEE Int. Conf. Commun. (ICC), London, UK, Jun. 2015, pp. 5985-5991.

[13] D. Shaviv, P. M. Nguyen, and A. Ozgur, "Capacity of the energyharvesting channel with a finite battery," IEEE Trans. Inf. Theory, vol. 62, no. 11, pp. 6436-6458, Nov. 2016.

[14] K. Tutuncuoglu, O. Ozel, A. Yener, and S. Ulukus, "The binary energy harvesting channel with a unit-sized battery," IEEE Trans. Inf. Theory, vol. 63, no. 7, pp. 4240-4256, Jul. 2017.

[15] R. Storn and K. Price, "Differential evolution - a simple and efficient heuristic for global optimization over continuous spaces," J. Glob. Optim., vol. 11, no. 4, pp. 341-359, Dec. 1997.

[16] M. Ivanov, F. Brannstrom, A. Graell i Amat, and P. Popovski, "Error floor analysis of coded slotted ALOHA over packet erasure channels," IEEE Commun. Lett., vol. 19, no. 3, pp. 419-422, Mar. 2015.

[17] Z. Sun, Y. Xie, J. Yuan, and T. Yang, "Coded slotted ALOHA for erasure channels: Design and throughput analysis," IEEE Trans. Commun., vol. 65, no. 11, pp. 4817-4830, Nov. 2017.

[18] F. Clazzer, E. Paolini, I. Mambelli, and C. Stefanovic, "Irregular repetition slotted ALOHA over the Rayleigh block fading channel with capture," in IEEE Int. Conf. Commun. (ICC), Paris, France, May 2017.

\footnotetext{
${ }^{7}$ Recall that we take the maximum degree of a repetition distribution as 8 .
} 